\title{
Verifying the Structure and Composition of Prepared Porous Catalytic Supports
}

\author{
Michael T.Y. Paul, ${ }^{1}$ Xin Zhang, ${ }^{1}$ Brenden Yee, ${ }^{1}$ Byron D. Gates ${ }^{1, *}$ \\ 1. Department of Chemistry and 4D LABS, Simon Fraser University, 8888 University Drive, Burnaby, \\ BC, V5A 1S6, Canada (email: bgates@sfu.ca).
}

The incorporation of catalytic nanoparticles (NPs) within nanostructured materials have been popular in recent years due to the excellent surface area to volume ratios that can be achieved for these materials and their ability to reduce the required loading of precious metal catalysts. The use of Pt and Pd NPs is especially attractive for power generating systems, such as proton exchange membrane and methanol oxidation fuel cells ${ }^{[1-3]}$. Further studies on these nanoparticle incorporated materials have revealed the underlying support materials can enhance catalytic properties and stabilities of the NPs ${ }^{[4,5]}$. However, due to processing constraints, loading of functional NPs is often limited to 2-D supports ${ }^{[7,8]}$. The incorporation of NPs into 3-D porous supports can be performed by sophisticated systems, such as physical vapor and atomic layer depositions ${ }^{[7-9]}$. In this study, we demonstrate a relatively simple and cost effective method, which was developed previously in our research group, for preparing various combinations of NPs loaded into 3-D structured materials ${ }^{[10-13]}$. The NP loading and spatial distribution were characterized by scanning electron microscopy (SEM), scanning transmission electron microscopy (STEM), and energy dispersive X-ray spectroscopy (EDS). Our technique could be used to prepare novel electrochemical and photochemical porous materials for increased catalytic efficiency and stability.

The method for preparing NPs loaded into a 3-D porous support involved the preparation of NP coated spherical polystyrene templates, self-assembly of these templates, infiltration with a support matrix, and removal of the sacrificial templates. The NPs ( $\mathrm{Pd}$ or $\mathrm{Pt}$ ) were mixed with the polystyrene templates at room temperature for $20 \mathrm{~min}$ to facilitate the first step of this process ${ }^{[10,12]}$. These decorated polymer spheres were purified to remove excess NPs, and self-assembled into thin films using either air-water interface self-assembly ${ }^{[10,13]}$, drop casting ${ }^{[12]}$, spin coating ${ }^{[14]}$, or vacuum filtration ${ }^{[15]}$. A support matrix was filled into the interstitial spaces between these sacrificial templates, and the polymer was then removed to produce nanostructured supports coated with NPs. This support matrix was deposited by either material specific electrochemical deposition ${ }^{[13]}$ or sol-gel techniques ${ }^{[15]}$. Templates were removed either by high temperature air oxidation $\left(>300^{\circ} \mathrm{C}\right.$ for $\left.>8 \mathrm{~h}\right)$ or room temperature digestion in organic or alkaline solutions ${ }^{[10-15]}$.

A typical SEM analysis of Pd NPs loaded onto the surfaces of Ni nano-bowls is shown in Figure 1A. Through the use of concentric backscattered (CBS) electron detection, the Pd NPs can be distinguished from the Ni support by the contrast according to differences in atomic (Z) number (Figure 1B). Loading of NPs is further confirmed with STEM based EDS mapping. We analyzed samples prepared with different loadings of Pt NPs supported by a $\mathrm{TiO}_{2}$ matrix. The EDS mapping indicated our ability to tune the loading of NPs on the sacrificial templates and to correlate this with loadings achieved within the porous support (Figure 2). Samples prepared with undiluted, 60\% diluted, and 90\% diluted concentrations of Pt NPs had corresponding Pt loadings of 1.6, 1.1, and 0.1 particles per $100 \mathrm{~nm}^{2}$, respectively.

These results demonstrated that 3-D NP decorated materials can be prepared with a tunable loading of 
noble metal NPs on a support prepared by sol-gel or electrodeposition techniques. The combination of microscopy techniques used in this study was imperative to verify the 3-D composition and structures of the materials. The analysis by SEM with a CBS detector provided a direct method to image these materials. The combination of STEM imaging and EDS analyses provided further verification of the material composition and spatial distribution (and loading) of the NPs. Additional investigations to be discussed include the preparation of cross-sections using focused ion beam (FIB) and lift-out techniques to further study the fine structures of the porous materials and the interface between the NPs and support materials that are imperative for electrochemical and catalytic applications.

References:

[1] O. Antoine, Y. Bultel, R.J. Durand, J. Electroanal. Chem. 10 (2010) p. 638.

[2] P. Waszczuk, J. Solla-Gullon, H.S. Kim, Y.Y. Tong et al, J. Catal. 203 (2001) p. 1.

[3] Z.P. Sun, X.G. Zhang, Y.Y. Liang et al, Electrochem. Comm. 11 (2009) p. 557.

[4] A.S. Eppler, J. Zhu, E.A. Anderson, G.A. Somorjai, Top. Catal. 13 (2000) p. 33.

[5] N. Toshima, T. Yonezawa, New J. Chem. 22 (1998) p. 1179.

[6] Z.Y. Zhang, L. Xin, K. Sun, W.Z. Li, Int. J. Hydrogen Energ. 36 (2011) p. 12686.

[7] T. Teranishi, M. Hosoe, T. Tanaka et al, J. Phys. Chem. B 103 (1999) p. 3818.

[8] M.H. Park, K. Kim, J. Kim et al, Adv. Mater. 22 (2010) p. 415.

[9] B. Lu, X. Li, T. Wang et al, Nano Lett. 13 (2013) p. 4182.

[10] B.K. Pilapil, M.C.P. Wang, M.T.Y. Paul et al, Nanotechnology 26 (2015) p. 055601.

[11] J. van Drunen, B. Pilapil, Y. Makonnen et al, ACS Appl. Mater. Interfaces 6 (2014) p. 12046.

[12] M.T.Y. Paul, B. Kinkead, B.D. Gates, J. Electrochem. Soc. 161 (2014) p. B3103.

[13] B. Kinkead, J. van Drunen, M.T.Y. Paul et al, Electrocatalysis 4 (2013) p. 179.

[14] P. Colson, R. Cloots, C. Henrist, Langmuir 27 (2011) p. 12800.

[15] A. Blanco, E. Chomski, S. Grabtchak et al, Nature 405 (2000) p. 437.

[16] This research was supported in part by the Natural Sciences and Engineering Research Council (NSERC) of Canada, the Canada Research Chairs Program (B.D. Gates), and CMC Microsystems through the MNT Financial Assistance program that facilitated access to materials characterization services. This work made use of 4D LABS shared facilities supported by the Canada Foundation for Innovation (CFI), British Columbia Knowledge Development Fund (BCKDF), Western Economic Diversification Canada, and Simon Fraser University.

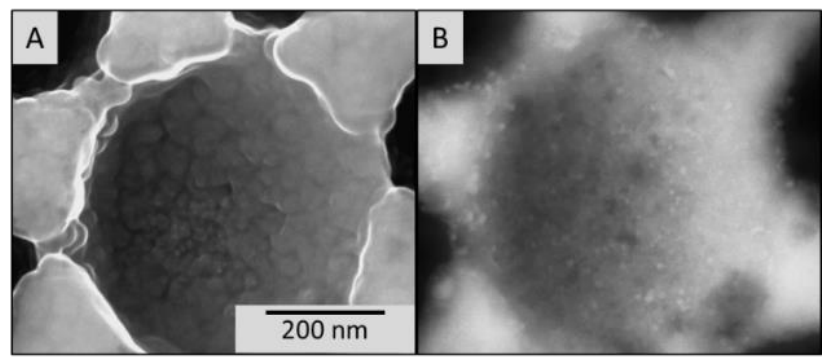

Figure 1. Scanning electron micrographs of $\mathrm{Pd}$ NPs decorated onto the surfaces of Ni nano-bowls as imaged under the field-free mode by: (A) an Everhart-Thornley electron detector; and (B) a concentric backscattered electron detector.

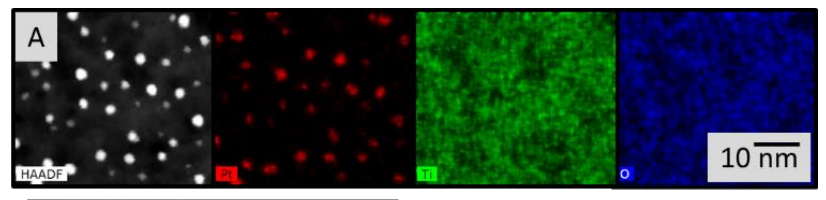

Figure 2. (A) Transmission electron microscopy high angle annular dark field (HAADF) image and $\mathrm{Pt}, \mathrm{Ti}$, and $\mathrm{O}$ EDS maps of $\mathrm{TiO}_{2}$ supported Pt NPs. HAADF image and Pt EDS maps of samples

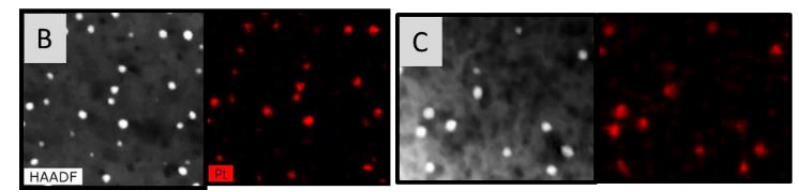
prepared from (B) $60 \%$ and (C) $90 \%$ less Pt NPs. 